\title{
Synthesis and Properties of Novel Non-Ionic Polyurethane Dispersion Based on Hydroxylated Tung Oil and Alicyclic Isocyanates
}

\author{
Xiaomin Yang1, Baixia Ren², Zhiyong Ren ${ }^{*}$, Lei Jiang1, Wentao Liu², Chengshen Zhu² \\ ${ }^{1}$ High \& New Technology Research Center of Henan Academy of Sciences, Zhengzhou, China \\ ${ }^{2}$ School of Materials Science and Engineering, Zhengzhou University Zhengzhou, China \\ Email: ${ }^{*}$ zyren23@163.com
}

Received November 2014

\begin{abstract}
Hydroxylated Tung oil (HTO) based nonionic polyurethane dispersion (HTO-NPUD) were synthesized using dicyclohexyl methane diisocyanate (HMDI) and HTO as main hydrophobic materials whereas polyethylene glycol-800 (PEG-800) as hydrophilic chain extender. To effectively study the effects of HTO on properties of NPUD, polypropylene glycol-400(PPG-400) based NPUD was prepared by HMDI reacting with PPG-400 and PEG-800. The structures of those novel nonionic polyurethane dispersions were characterized by FTIR and ${ }^{1}$ H NMR. Moreover, particle size and size distribution, cloud point and surface tension had been investigated. Results showed that, by comparing with PPG based NPUD (PPG-NPUD), the introduction of HTO into NPUD result in larger particle size and more uniformed particle size distribution, higher cloud point and lower surface tension.
\end{abstract}

\section{Keywords}

Nonionic Polyurethane, Hydroxylated Tung Oil, Cloud Point, Surface Tension

\section{Introduction}

Waterborne polyurethanes or polyurethane dispersions (PUDs) are a class of polymer dispersions with excellent comprehensive performance which exhibit a wide range of advantages such as low volatility of organic solvent, non-toxic, non-flammability and energy saving. It is for this reason that PUDs have been gaining thorough study and will be further developed into more environmental, biodegradable and functional materials. Among all types of PUDs, nonionic PUD (NPUD) without ion dangling on its molecular chain is provided with improved acid and alkali resistance, electrolyte sexual tolerance and can be applied in the development of synthetic leather surfactant and textile finishing agent [1]-[3].

\footnotetext{
${ }^{*}$ Corresponding author.
}

How to cite this paper: Yang, X.M., Ren, B.X., Ren, Z.Y., Jiang, L., Liu, W.T. and Zhu, C.S. (2015) Synthesis and Properties of Novel Non-Ionic Polyurethane Dispersion Based on Hydroxylated Tung Oil and Alicyclic Isocyanates. Journal of Materials Science and Chemical Engineering, 3, 88-94. http://dx.doi.org/10.4236/msce.2015.31013 
Tung oil [4], one of Chinese specialty, is a kind of renewable, environmentally friendly and biodegradable natural resource with attainable development. The introduction of tung oil related structure which contains 3 conjugated double bonds per fatty acid chain [5] into polyurethane can not only make it more eco-friendly but also provide potential functions such as being oxidized, hybridization with other materials or cross-link in a complex molecule. These features make it become an industrially attractive raw material for the polyurethane industry. Before preparing polyurethane, tung oil is required to be functionalized [1]. Having both triglyceride and unsaturated bond as active points, tung oil could be hydroxylated by aminolysis or alcoholysis from ester and/or by reaction at the double bond [6] [7]. HTO-based polyols prepared by the first method not only introduce hydroxy groups but also retain the active conjugated double bonds in its molecular chain. Those hydroxylated by the latter method destroy double bonds forming polyhydroxy (three or more hydroxy) compounds which are usually suitable for polyurethane foam. Moreover, the functionality of hydroxy group is hard to control.

The HTO prepared by first method (through ester) has functionality of two and can be reacted with isocyanate. This passage aims to synthesize a novel HTO-based NPUD used for macromolecule surfactant with conjugated double bonds. Compared with micromolecule surfactants, whose residues after emulsification may harm the polymer matrix, macromolecule surfactants have properties of perfect dispersity, film-forming ability, environmental friendliness, stability [8] [9] and, furthermore, the ability of forming polymer alloy by blending or crosslinking with the polymer matrix. And properties of those polymer alloys could be improved owing to the potential ability of double bond provided by tung oil. Therefore, the investigation on such HTO-NPUD broadens the application of macromolecule surfactant, lessens the cost of raw material and better the environment.

The present study aims to develop novel, biobased HTO-NPUD with post crosslinking function, based on the HTO that is hydroxylated via ester mentioned above. Firstly, NPUD was synthesized from HMDI reacting with HTO and PEG-800. Then, the structures of the NPUDs were characterized by IR and NMR, and the influence of HTO on the performance of NPUD were studied and analyzed including particle size, cloud points and surface tension. In order to make better investigation analysis for effect of HTO on PUD, PPG-NPUD was selected to compare with HTO-NPUD. The reason to use PPG-NPUD as comparison is that PPG-400 is a kind of common material in synthesis of polyurethane and provides similar molecular weight with HTO.

\section{Experimental Section}

\subsection{Materials}

Polyethylene glycol-800 (PEG-800, Bayer, Germany), polypropylene glycol-400 (PPG-400, Haian petrochemical plant, China). Reagents above were dealt with vacuum drying oven at certain temperature before use. HTO was kindly provided from US A-line Company. Dicyclohexyl methane diisocyanate (HMDI) was purchased from Wanhua Chemical Group Company. N-methyl pyrrolidone (NMP) was purchased from Shanghai Chemical Reagent Company of China Pharmaceutical Group, freed from moisture using anhydrous 4A molecular sieve. Distilled water was used as the dispersing phase.

\subsection{Synthesis of NPUD}

The recipes for NPUD are showed in Table 1. PEG-800, PPG-400 and NMP have been dewatered in advance.

HTO, PEG and HMDI were simultaneously added into a $500 \mathrm{~mL}$ four-necked flask equipped with stirrer and thermometer. The reaction was carried out in the protection of nitrogen gas and under a constant temperature at $80^{\circ} \mathrm{C}-90^{\circ} \mathrm{C}$ heating with oil bath. When the $\mathrm{NCO}$ group content reached to a designed value, distilled water was added and the stirring rate was raised after the temperature was lowered. Then HTO-NPUD was obtained by the

Table 1. The basic recipes, molecular weight and cloud point of two NPUDs.

\begin{tabular}{cccccccc}
\hline \multirow{2}{*}{ Samples } & \multicolumn{9}{c}{ The Basic Recipes } & & \multicolumn{2}{c}{$\mathrm{M}_{\mathrm{n}}(\mathrm{g} / \mathrm{mol})$} & $\begin{array}{c}\text { Cloud Point } \\
\left({ }^{\circ} \mathrm{C}\right)\end{array}$ \\
\cline { 2 - 5 } & PPG-400 & HTO & PEG-800 & HMDI & & 34 \\
PPG-NPUD & 1 & 0 & 1 & 2 & 7800 & 69 \\
HTO-NPUD & 0 & 1 & 1 & 2 & 8200 & 69 \\
\hline
\end{tabular}


emulsification of HTO-NPU prepolymer. In this reaction process, a small amount of NMP was added to adjust the viscosity. The synthesis of PPG-NPUD was similar to that of HTO-NPUD. The synthesis process of NPUDs is showed in Figure 1.

\subsection{Characterization}

All infrared spectra of NPUDs were obtained using a SHIMADZU FTIR-8700 Spectrophotometer. The frequency range covered was from 4000 to $400 \mathrm{~cm}^{-1}$ by averaging 16 scans at a resolution of $4 \mathrm{~cm}^{-1}$. ${ }^{1} \mathrm{H} \mathrm{NMR}$ spectra were recorded on a Bruker Avance $\quad$ III00MH superconducting NMR Spectrometer using DMSO-d6 as the solvent at a concentration of $\sim 5 \%(w / v)$. All spectra were recorded at room temperature (298 K). Chemical shifts $(\delta)$ are given in parts per million with tetramethylsilane (TMS, 0.1\%) as internal standard. Coupling constants $(\mathrm{J})$ are given in Hertz.

The average particle size and distributions of NPUDs were measured with a Malvern ZEN3690 laser particle sizer (UK). The cloud points of aqueous polyurethane were measured using distilled water measurement according to GB/T5559-1993. Samples were regularly diluted to different concentrations and the surface tensions were investigated with DATAPHYSICS DCAT21 automatic interface tensiometer.

\section{Results and Discussion}

\subsection{Characterization of Nonionic Polyurethane}

The FTIR spectra of PPG-NPUD and HTO-NPUD are shown in Figure 2. The peaks found at $1538 \mathrm{~cm}^{-1}(1529$ $\mathrm{cm}^{-1}$ ) and $1247 \mathrm{~cm}^{-1}$ belong to Amid II and Amid III bands formed by vibrational coupling between N-H deformation vibration and C-N stretching vibration [10], respectively. Peak at $1650 \mathrm{~cm}^{-1}$ belongs to Amid I band.

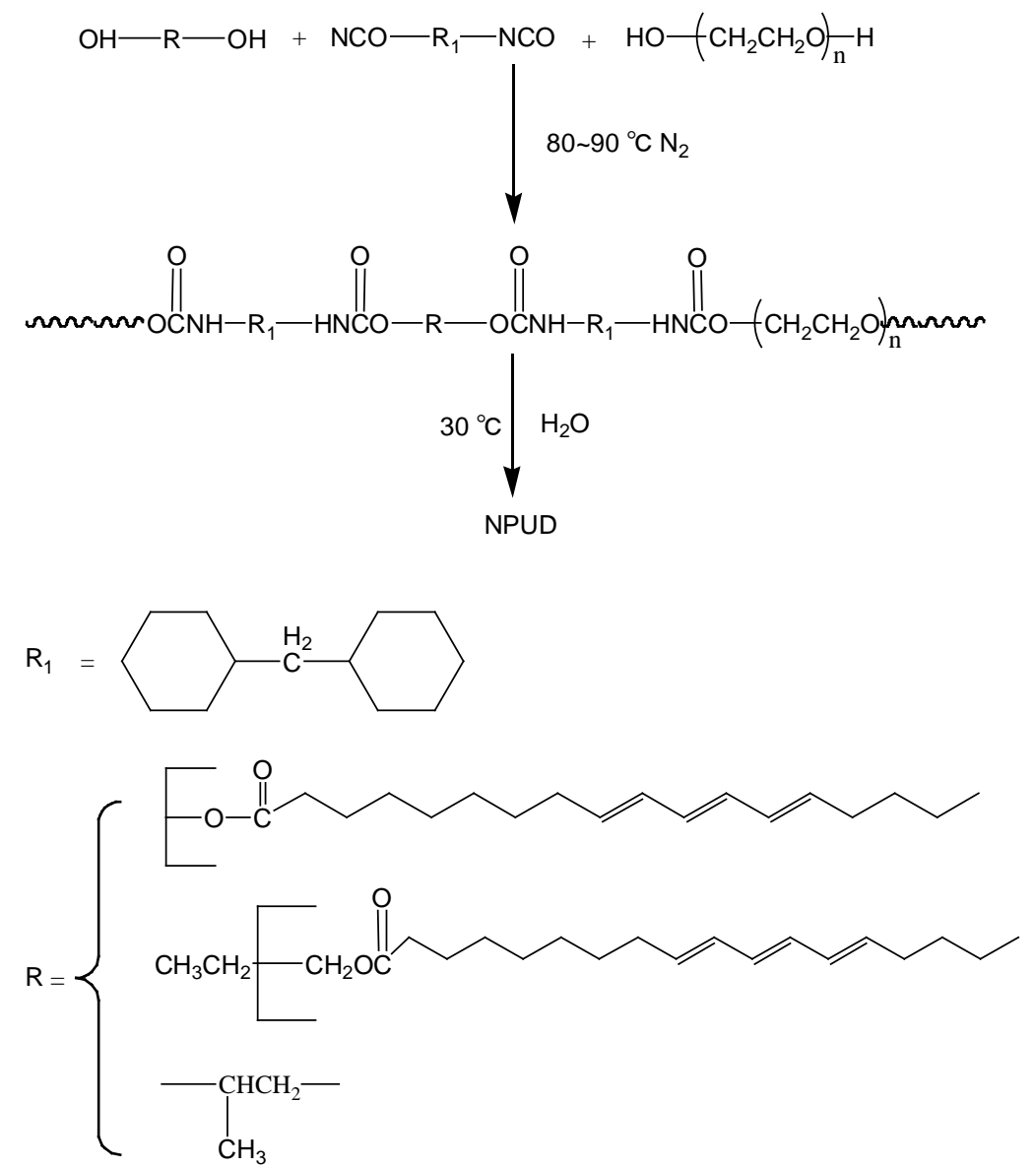

Figure 1. Synthesis process of NPUDs. 


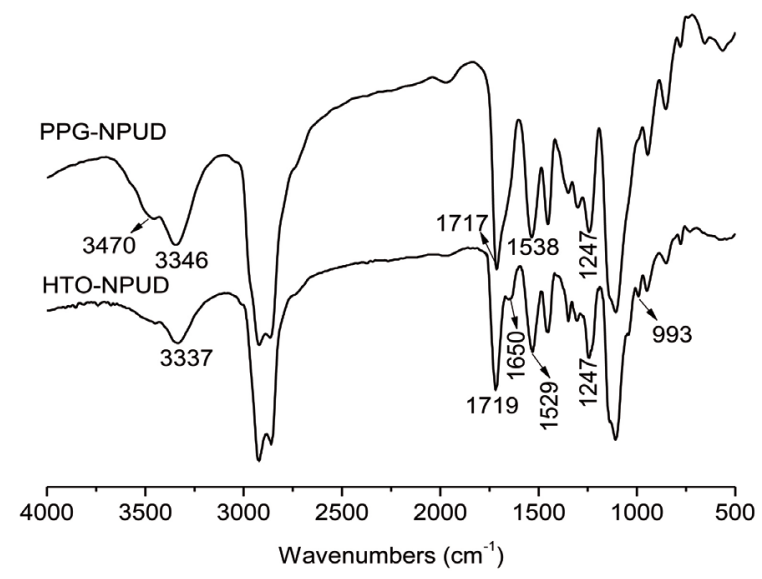

Figure 2. The FTIR spectra of PPG-NPUD and HTO-NPUD.

The absorption peak at $1110 \mathrm{~cm}^{-1}$ are due to hydrophilic C-O-C stretching vibration. No peak at around 2250 $\mathrm{cm}^{-1}$, which is character peak of free NCO group, indicates that NCO in this product has totally reacted. The above characteristic spectra prove that the resultants are the expected polyurethanes. The absorption peaks of $\mathrm{N}-\mathrm{H}$ at $3346 \mathrm{~cm}^{-1}, 3337 \mathrm{~cm}^{-1}$ illustrate that $\mathrm{N}-\mathrm{H}$ possibly formed hydrogen bond with $\mathrm{C}=\mathrm{O}$. And this phenomenon is proved by the fact that the absorption peaks of $\mathrm{C}=\mathrm{O}$ are at $1719 \mathrm{~cm}^{-1}$ and $1717 \mathrm{~cm}^{-1}$ instead of $1732 \mathrm{~cm}^{-1}$ which is the characteristic peak of free $\mathrm{C}=\mathrm{O}$. In comparison with PPG-NPUD, there is one more peak at 993 $\mathrm{cm}^{-1}$ that represents characteristic peak of conjugated double bond in the spectrum of HTO-NPUD, suggesting that HTO is successfully introduced into NPUD structure.

The ${ }^{1} \mathrm{H}$ NMR spectra of PPG-NPUD and HTO-NPUD are presented in Figure 3. The peaks at $6.80-7.25$ ppm belong to proton $\mathrm{NH}$ of -NH-COO- group. Proton $\mathrm{CH}_{2}$ joined with oxygen located at $3.15-3.71 \mathrm{ppm}$. Peaks at $1.56-1.75 \mathrm{ppm}$ and $0.88-1.75 \mathrm{ppm}$ are related to proton $\mathrm{CH}_{3}$ and proton $\mathrm{CH}_{2}$. The small peaks displayed at 5.0 - 6.5 ppm in Figure 3(b) are attributed to three conjugated double bonds. These evidences also prove that the synthesis of NPUD and the introduction of HTO into NPUD are successful. Moreover, both hydrophilic group and hydrophobic chain segment are proved to be introduced into NPUD.

\subsection{Particle Size and Size Distribution of NPUD}

PUD is macromolecule of polyurethane dispersing in water in the form of latex particle whose diameter is particle size. Particle size and its distribution have a direct effect on the emulsion's properties such as the color, transparency and stability. The decrease in particle size will result in an increase in transparency, stability [11] and dissolution rate [12] of PUD which is a kind of milky white emulsion.

It is evident from Figure 4 that the introduction of HTO makes a significant difference in particle size and size distribution compared with PPG-NPUD. For PPG-NPUD, most particle diameters are 2 - $10 \mathrm{~nm}$, others distribute between $80-120 \mathrm{~nm}$, indicating that the size distribution of PPG-NPUD is a multiple distribution. These two size distributions may be induced by the following reason. It is expected that the reaction of oleophyllic HMDI and PPG forms oleophyllic particles with large diameter. While reacting rate of HMDI and hydrophilic PEG is higher than that of HMDI and PPG, resulting in another smaller particle size. By contrast, particle size of HTO-NPUD is relatively larger and size distribution is narrower (most in $5-100 \mathrm{~nm}$ and few in $2-10 \mathrm{~nm}$ ). The possible reason is that, compared with PPG-NPUD which is a linear chain polymer containing hydrophilic group, HTO was introduced into HTO-NPUD in the form of hydrophobic side chain, creating intermolecular winding then causing larger particle size. Meanwhile, the space steric hindrance of the large side chain weakens the hydrogen bonding interaction inside the NPU, balancing hydrogen bonding effect on the whole system then resulting in more uniformed particle size distribution.

\subsection{Cloud Point}

The cloud point is a temperature at which a homogenous surfactant solution separates into two coexisting phases. It is basically affected by its molecular structure [13], its own concentration [14] and the presence of additives [15]. For nonionic surfactant which hydrogen bond can be formed with water, the intensity and number of 


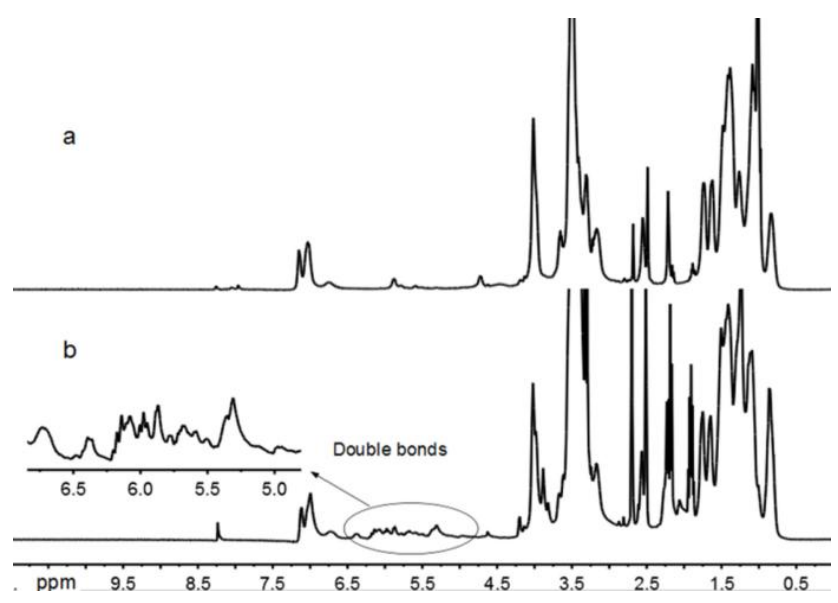

Figure 3. The ${ }^{1} \mathrm{H}-\mathrm{NMR}$ spectra of NPUD ((a): PPG-NPUD; (b): HTO-NPUD).

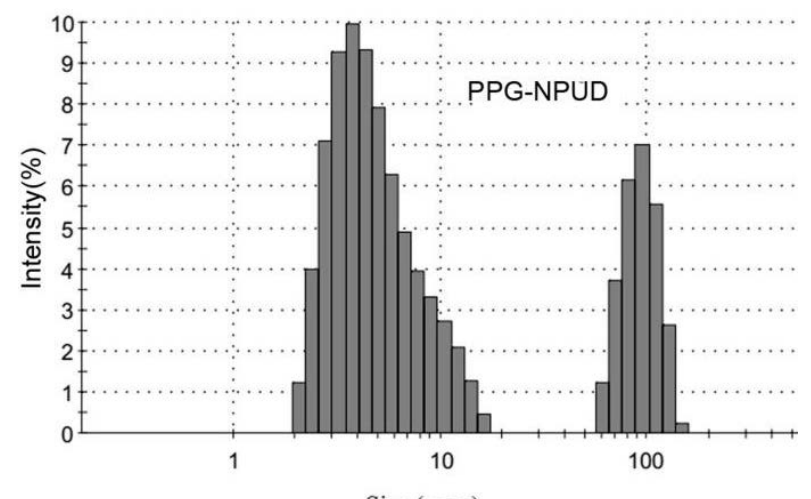

Size (r.nm)

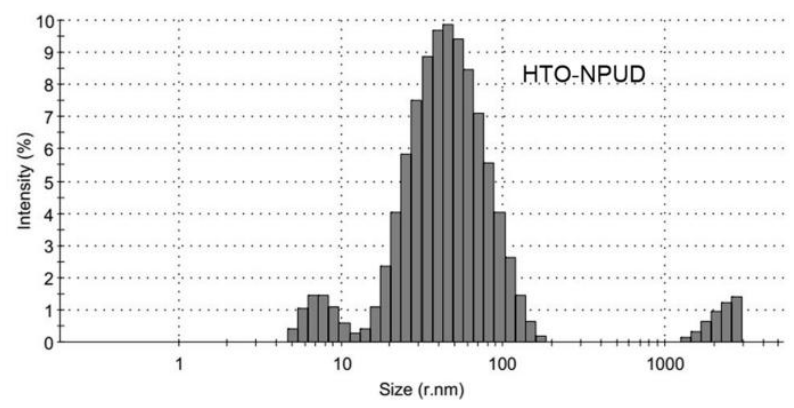

Figure 4. The particle sizes and distributions of PPG-NPUD and HTO-NPUD.

hydrogen bonds have profound impact on cloud point [16] as well.

As is indicated in Table 1, the cloud point of HTO-NPUD is much higher than that of PPG-NPUD, which shows that the introduction of HTO structure increases the cloud point of NPUD. According to the influence of HTO on particle size and size distribution of NPUD, the hydrogen bonding interaction inside the polymer is weakened, while the intensity and number of hydrogen bonds between NPUD and water are enhanced. So cloud point increases with increasing amount of energy consumed to destroy those hydrogen bonds between NPU and water.

\subsection{The Surface Tension}

The surface tension is induced by the liquid surface shrinkage that is an important property of surfactant. Figure 5 shows that the surface tension of HTO-NPUD $(45.6 \mathrm{mN} / \mathrm{m})$ is much lower than that of PPG-NPUD $(55.8 \mathrm{mN} / \mathrm{m})$ 


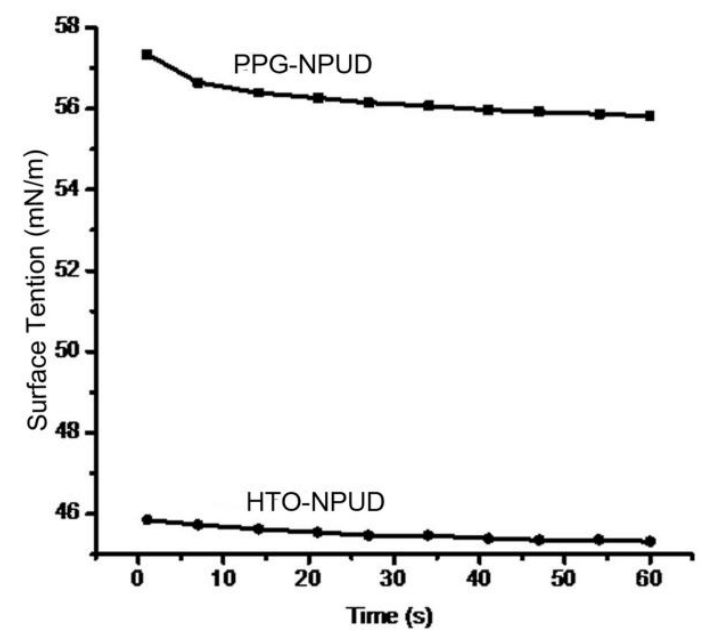

Figure 5. The surface tension changes of PPG-NPUD and HTO-NPUD with time.

in the same conditions, which indicates that the surface tension of NPUD is significantly affected by the introduction of HTO. On the surface of NPUD aqueous emulsion, hydrophilic groups combine with water inside, while hydrophobic chains arrange outside, whose repulsion destroy the power among water molecules. So the surface tension reduction may be induced by the introduction of long oleophyllic alkyl chain as side chain of HTO into NPUD.

\section{Conclusion}

A type of novel NPUDs have been successfully synthesized by the reaction of HMDI with HTO/PPG-400 and PEG-800. HTO with three conjugated double bonds had been introduced into the NPUD. In spite of the fact that the particle size of HTO-NPUD was much larger than that of PPG-NPUD, the introduction of HTO may improve the stability of NPUD emulsion owing to the more uniformed particle size distribution. The cloud point of HTO-NPUD was remarkably higher than that of PPG-NPUD, indicating that HTO significantly improved thermal stability of NPUD. Furthermore, the surface tension of the emulsion was reduced by the introduction of hydrophobic chain from HTO. The lower surface tension suggested that HTO-NPUD had obvious surface active property and provided possibility of using HTO-NPUD as surfactant. And the three conjugated double bonds would endow more excellent performance that is worth to be further studied.

\section{Acknowledgements}

This work is supported by the National Nature Science Foundation (21074030).

\section{References}

[1] Lligadas, G., Ronda, J.C., Galià, M. and Cádiz, V. (2006) Novel Silicon-Containing Polyurethanes from Vegetable Oils as Renewable Resources. Synthesis and Properties. Biomacromolecules, 7, 2420-2426. http://dx.doi.org/10.1021/bm060402k

[2] Guo, Y.S., Ma, X.Y., Wu, Z., Zhang, S.F. and Yang, X.P. (2013) Synthesis and Modified of Nonionic Waterborne Polyurethane for Synthetic Leather. China Leather, 42, 47-57.

[3] Naghash, H.J., Iravani, M. and Akhtarian, R. (2014) Synthesis and Characterization of Monoallyl-End-Capped Diethylene Oxide-Based Polyurethane Surfactant. Synthesis and Reactivity in Inorganic Metal-Organic and Nano-Metal Chemistry, 44, 927-934. http://dx.doi.org/10.1080/15533174.2013.790441

[4] Ren, Z.Y., Zhang, C.L., Wang, H.F., Zhao, W., Fu, Y. and Zhang, Y.B. (2013) Facile Synthesis and Newly Found Biological Activity of Aminolyzedtung Oil Components with Conjugated Double Bonds. RSC Advances, 3, 17712-17716. http://dx.doi.org/10.1039/c3ra42860e

[5] Meiorin, C., Mosiewicki, M.A. and Aranguren, M.I. (2013) Ageing of Thermosets Based on Tung Oil/Styrene/Divinylbenzene. Polymer Testing, 32, 249-255. http://dx.doi.org/10.1016/j.polymertesting.2012.10.009 
[6] Silva, V.R., Mosiewicki, M.A. and Yoshida, M.I. (2013) Polyurethane Foams Based on Modified Tung Oil and Reinforced with Rice Husk Ash I: Synthesis and Physical Chemical Characterization. Polymer Testing, 32, 438-445. http://dx.doi.org/10.1016/j.polymertesting.2013.01.002

[7] Ren, Z.Y., Jiang, L. and Fu, Y. (2012) Preparation Method of Hydroxylation Tung Oil and Aminolysis of It's Ester Group. CNPatent, No. 101845367A.

[8] Zhang, M.Y., Chen, J.J. and Yang, L.C. (2011) Research on Synthesis and Application of Anti-wrinkle Finishing Agent of Aliphatic Nonionic Waterborne Polyurethane. PU Technology, No. 9, 70-73.

[9] Zheng, J., Luo, J.X. and Zhou, D.W. (2010) Preparation and Properties of Non-ionic Polyurethane Surfactants. Colloids and Surfaces A: Physicochemical and Engineering Aspects, 363, 16-21. http://dx.doi.org/10.1016/j.colsurfa.2010.04.001

[10] Bellamy, L.J. (1980) The Infrared Spectra of Complex Molecules. 2nd Edition, Chapman and Hall Ltd., London. http://dx.doi.org/10.1007/978-94-011-6520-4

[11] Zhang, H.T., Liu, Y.P. and Li, X.J. (2011) Double Latex Particle Size Distribution of Concentrated Inverse Polymer Emulsion and Its Correlation with Stability. Actapolymericasinica, 8, 832-837.

[12] Mosharraf, M. and Nyström, C. (1995) The Effect of Particle Size and Shape on the Surface Specific Dissolution Rate of Microsized Practically Insoluble Drugs. International Journal of Pharmaceutics, 122, 35-47. http://dx.doi.org/10.1016/0378-5173(95)00033-F

[13] Huibers, P.D.T., Shah, D.O. and Katritzky, A.R. (1997) Predicting Surfactant Cloud Point from Molecular Structure. Journal of Colloid and Interface Science, 193, 132-136. http://dx.doi.org/10.1006/jcis.1997.5053

[14] Koshy, L., Saiyad, A.H. and Rakshit, A.K. (1996) The Effects of Various Foreign Substances on The Cloud Point of Triton X 100 and Triton X 114 Colloid Polym. Colloid and Polymer Science, 274, 582-587. http://dx.doi.org/10.1007/BF00655234

[15] Li, J.L., Bai, D.S. and Chen, B.H. (2009) Effects of Additives on the Cloud Points of Selected Nonionic Linear Ethoxylatedalcohol Surfactants. Colloids and Surfaces A: Physicochemical and Engineering Aspects, 346, 237-243. http://dx.doi.org/10.1016/j.colsurfa.2009.06.020

[16] Mu, J.H., Li, G.Z. and Liu, S.J. (1999) Effect of Additives on the Cloud Point of Nonionic Surfactant. Detergent \& Cosmetics, S1, 14-16+35. 